\title{
Ghondroblastie Osteosarcoma of Jaw Bone
}

\section{Khadse Smita Vasudeo}

From the Department of Oral Pathology and Microbiology, Maharaja Ganga

Singh Dental College and Research Centre, Sri Ganganagar, Rajasthan, India.

\section{Abstract:}

Osteosarcoma is a malignant mesenchymal tumor whose cancerous cells produce osteoid matrix. It is the common primary malignant bone tumor, but only $4-7 \%$ of the osteosarcomas occur in the jaws. Considerable histopathologic variability was seen in the jaw osteosarcoma. Here a case of chondroblastic osteosarcoma of jaw with their clinical, radiographic and histological findings is presented.

Key words: Osteosarcoma, Bone Neoplasms, Liver Neoplasms, Mesenchymoma.

\section{Introduction}

Osteosarcoma is a primary malignant tumor of bone of mesenchymal tissues that histopathologically shows osteoid formation. Head and neck osteosarcoma is infrequent and usually presents in the third and fourth decades of life [1]. Gnathic osteosarcoma has some biological aspects different from osteosarcoma of long bones, such as older age at presentation, longer survival rate, rare metastases and local recurrence difficult to control [2]. Jaw osteosarcoma shows considerable histopathologic variability. Depending on the relative amounts of osteoid, cartilage or collagen fibers produced by the tumor, it is subclassified into the osteoblastic, chondroblastic and fibroblastic types [3]. A case of chondroblastic osteosarcoma is being reported and various aspects of chondroblastic variant are discussed

\section{Case Report}

A 35 year old male patient reported with the complaint of a swelling on upper left jaw since last 2 and $1 / 2$ months. The swelling was initially small and present on left palate, gradually increased buccally and palatally to achieve size of approximately $8 \times 5 \mathrm{~cm}$. Swelling was associated with pain which was continuous, non-radiating and aggravated on yawning and eating. The patient denied any history of trauma. In tissue abuse habits, he had habit of chewing tobacco 5-6 times a day since 2 months and used to keep the tobacco quid in lower anterior vestibule. He was using mishri and finger for cleaning his teeth. General medical and surgical history was noncontributory. Extraorally, swelling extended superoinferiorly from left inferior orbital margin to the line joining left corner of mouth and the left tragus of ear and anteroposteriorly from the right

Corresponding Author: Dr. Khadse Smita Vasudeo

Email: dr_smita@rediffmail.com

Received: December 24, 2013 | Accepted: December 25, 2013 | Published Online: December 30, 2013 This is an Open Access article distributed under the terms of the Creative Commons Attribution License (creativecommons.org/licenses/by/3.0)

Conflict of interest: None declared | Source of funding: Nil | DOI: http://dx.doi.org/10.17659/01.2013.0110 
corner of mouth to $2 \mathrm{~cm}$ anterior to the left tragus of ear. The swelling was irregular, diffuse, hard in consistency and was of the same colour as adjacent skin [Fig. 1]. Both submandibular lymph nodes were mobile, palpable and non-tender.

Intra-oral examination revealed firm, irregular, pinkish mass of approximately $8 \times 5 \mathrm{~cm}$, extending buccally from 13 to 27 , and palatally involved the whole left hard palate and crossing the midline by $1 \mathrm{~cm}$, with distinct margin at places [Fig.2,3]. Examination of teeth revealed grade II mobility with all teeth in upper left quadrant.

Computed tomography (CT) showed left maxillary mass extending to left nasal cavity. It also showed bony destruction and right deviation of nasal septum [Fig.4]. After thorough clinical and radiological examination, tumor was resected by hemimaxillectomy. On gross examination, the excised biopsy specimen consisted of lobulated mass that measured $9 x 7 \mathrm{~cm}$, was firm in consistency and reddish pink in colour [Fig.5]. The cut surface was grayish white in colour with glistening appearance.

Hematoxylin and eosin stained tissue sections showed tumor tissue comprised of highly cellular cartilagenous tissue [Fig.6], chondrocytes in lacunae arranged in lobular pattern, with pleomorphic \& hyperchromatic nuclei [Fig.7] and osteoid areas with osteoblastic rimming, surrounded by malignant cells. At some places dilated blood vessels along with hemorrhagic areas were seen.

On the basis of clinical, radiological and histopathological features a diagnosis of "Chondroblastic osteosarcoma of left maxilla" was given.

\section{Discussion}

Osteosarcoma is one of the commonest skeletal neoplasm, but seen occasionally in the jaw bones,

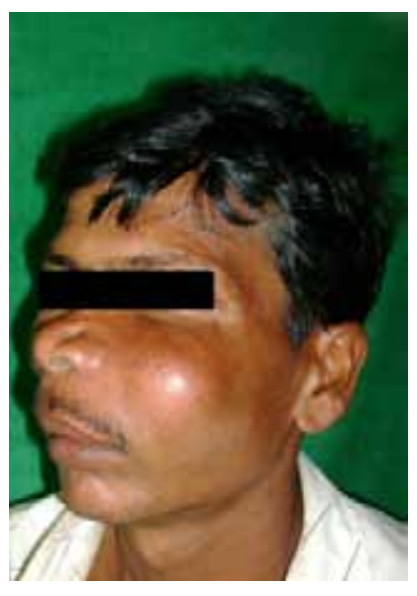

Fig.1: A diffuse swelling on left maxillary region of approximately $8 \times 5 \mathrm{~cm}$ and deviation of nasal septum.

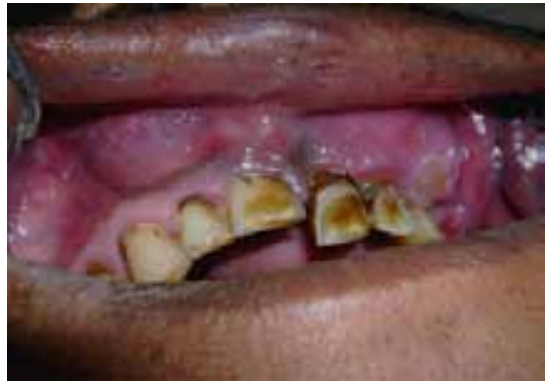

Fig.2: Reddish pink growth extending from 1327 , obliterating the buccal sulcus.

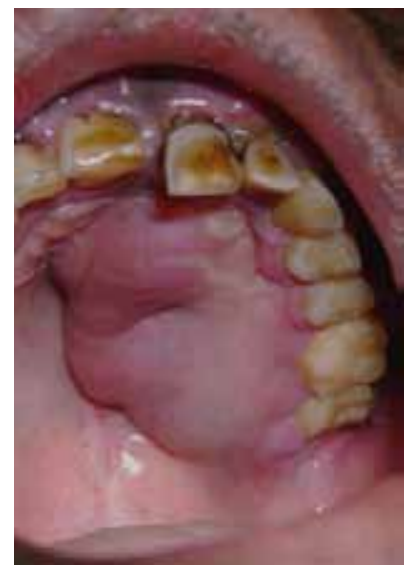

Fig.3: Irregular pinkish growth on left side of hard palate. 
approximately 4-7\% [1]. Osteosarcomas of gnathic bones usually involve adults in third and fourth decades of life. In this paper, osteosarcoma is being reported in 35 years male. According to Slootweg and Muller, older patients have better prognosis due to increased resistance to the tumor [4]. Men are affected slightly more frequently than women, which may reflect the longer period of skeletal growth and the additional volume of bone present in the male. However, mandibular tumors are more prevalent in the women [5].

Maxilla and mandible are involved with almost equal frequency. Mandibular tumors arise more frequently in the symphysis, whereas maxillary tumors are discovered more commonly in the alveolar ridge, sinus floor, and palate [6]. In our case report, similar site involvement is seen.

Several epidemiological risk factors have been related to the development of osteosarcoma, including a history of ionizing radiation exposure, fibrous dysplasia, retinoblastoma or history of trauma [7]. Risk factors were negative with our case.

No single feature on radiographs is diagnostic. Osteosarcomatous lesion can be purely osteolytic, purely osteoblastic, or a mixture of both. CT scan of the primary lesion helps in delineating the location and the extent of the tumor and is critical for surgical planning. In our case, CT scan shows left maxillary mass obliterating the sinus and destroying palatal, orbital and nasal wall and right deviation of nasal septum. CT of the chest is more sensitive than plain radiography for assessing pulmonary metastases. MRI of the primary lesion is the best method for assessing the extent of intramedullary disease. MRI findings are best correlated with the extent of disease assessed at the time of definitive surgery [8]. Chondroblastic osteosarcoma is defined as: any osteoid and bone/or bone that is produced by uncommitted mesenchymal cell without first passing through a cartilaginous phase, no matter what the

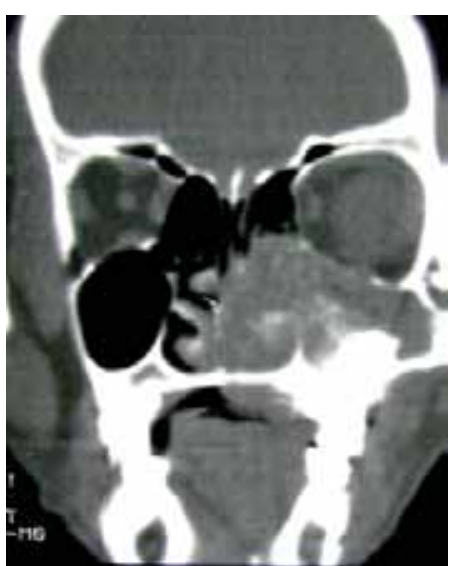

Fig.4: CT scan showing exophytic lytic lesion involving left maxillary sinus and extending into the nasal cavity.

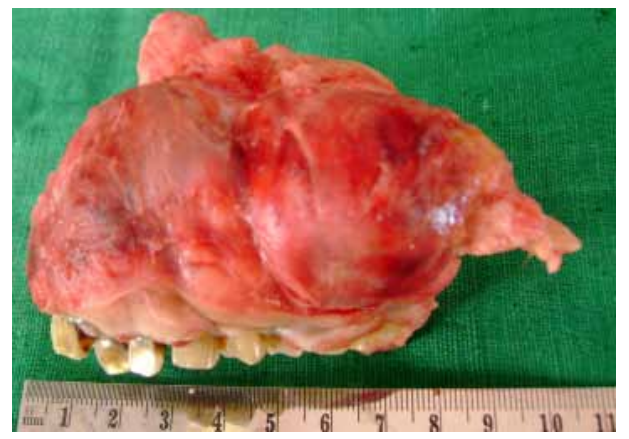

Fig.5: Gross specimen showing reddish pink lobulated mass of $9 \times 7 \mathrm{~cm}$ along with resected maxilla.

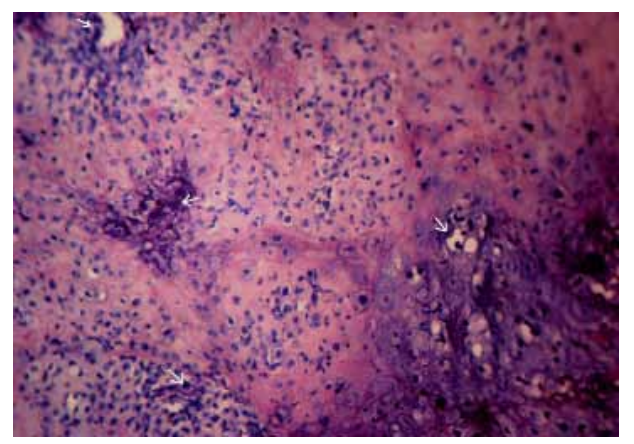

Fig.6: Highly cellular cartilagenous tissue $(H \& E$, 100X) 
extent of the chondromatous element [8].

The presence of osteoid is the distinguishing feature of chondroblastic osteosarcoma, but osteoid may be absent in small, unrepresentative biopsy specimens. The diagnosis of chondroblastic osteosarcoma may not be possible on the basis of clinical examination and roentgenographic investigation every time. Biopsy for histological identification is essential before definitive therapy is undertaken. A recently found gene encoding an intranuclear osteocalcin promoter - "cbfal" appears to be a potential marker in the definitive diagnosis of malignant bone tumors. In addition, the detection of alkaline phosphatase activity in imprint preparations obtained from the cut surface of osteosarcoma before fixation, is regarded as diagnostic of osteosarcoma, when used in combination with radiographs $[3,9,10]$. Chondrosarcoma is negative for this enzyme.

Chondrosarcoma of the jaws is extremely uncommon. It occurs primarily in the anterior maxilla. Although chondrosarcoma occurs in patients of all ages, most of those affected are over 50 years of age [11]. Potdar found 14 chondrosarcomas, equally divided in the maxilla and mandible [9]. Histopathologically, chondrosarcomas have wide range of presentations, from well differentiated growths resembling benign cartilage tumors to high grade malignant lesion [7].

Osteosarcoma of the jaws and facial bones are more common than chondrosarcomas, out of this chondroblastic variety accounts for 50\% [1]. On the basis of the predominant component of the stroma, lesions can be subtyped, Kragh and Dahlin designated $14(32 \%), 22(50 \%)$, and 8 $(18 \%)$ cases as osteoblastic, chondroblastic and fibroblastic osteosarcomas, respectively, in their series of 44 patients of osteosarcomas of the jaws and facial bones. This is in contrast to $50 \%, 27 \%$, and $23 \%$, respectively for osteosarcomas of other bones [12].

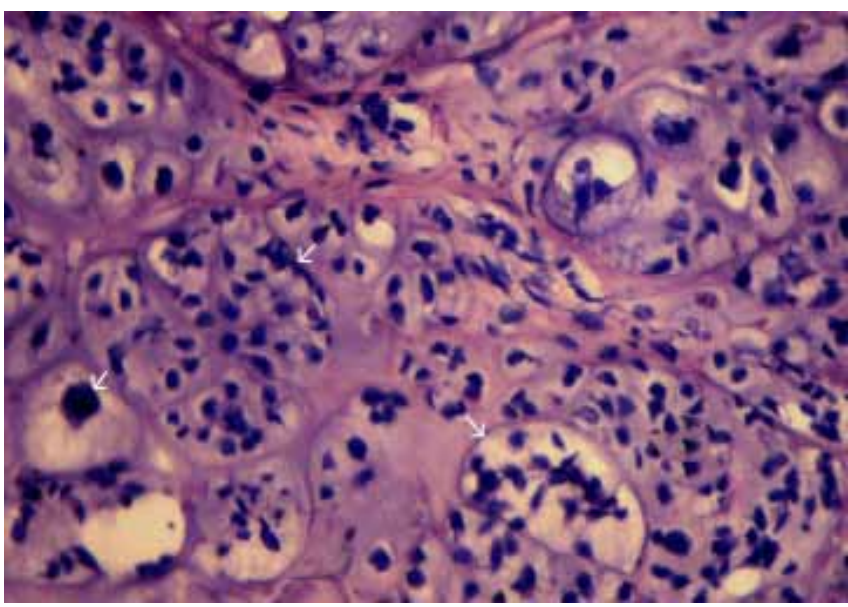

Fig.7: Chondrocytes in lacunae arranged in a lobular pattern, with pleomorphic and hyperchromatic nuclei (H \& E 400X).

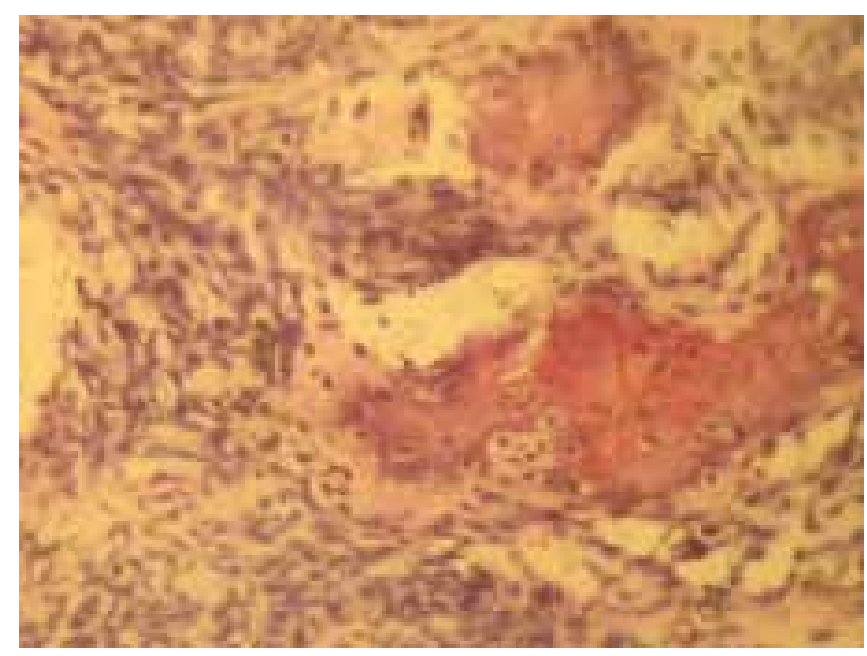

Fig.8: Bone and osteoid tissue surrounded by malignant cells (H \& E 400X).

According to Garrington's series of 45 cases, the overall 5 year survival rate for maxillary osteosarcoma was $25 \%$ and for mandibular osteosarcoma, $41 \%$. There was no correlation between histological characteristics of the tumor and prognosis [7]. However, according to Bennett et al, chondroblastic variant has better prognosis than others [10]. In case of chondrosarcoma, the 
overall 5 year survival rate is approximately $90 \%$ for grade I lesions, $81 \%$ for grade II lesions and $43 \%$ for grade III lesions [11]. Chondroblastic osteosarcoma is sensitive for chemotherapy and radiotherapy while chondrosarcoma is resistant for it. Follow-up for 6 months show no recurrence.

\section{Conclusion}

Appropriate diagnosis of chondroblastic variant of osteosarcoma and its differentiation from chondrosarcoma is of great help to expose the wolf in sheep clothing.

\section{References}

1. Lucas RB. Tumours of bone. In: Lucas RB, Pathology of tumours of the oral tissues. $4^{\text {th }}$ edition. Edinburgh:Churchill Livingstone;1984;197-200.

2. August $M$, Magennis, Dewitt D. Osteosarcoma of the jaws: factors influencing prognosis. Int J Oral Maxillofac Surg. 1997;26:198-204.

3. Baghaie F, Motahhary P. Osteosarcoma of the jaws: a retrospective study. Acta Medica Iranica. 2003;41:113-121.

4. Soares RC, Soares AF, Souza LB, Dos Santos ALV, Pinto LP. Osteosarcoma of mandible initially resembling lesion of dental periapex: a case report. Braz Journ of Otorhinolaryngology. $2005 ; 71: 242-245$.

5. Altuwairgi $O$, Papagoerge MB, Karp DD.
Maxillary chondroblastic sarcoma: Presentation of two cases and a literature review. J Oral Maxillofac Surg.1996;54:1357-1364.

6. Smith Ac, Harvey L. Chondroid sarcoma of the maxilla. Int J Oral Maxillofac Surg. 1987; 16:232-235.

7. Rajendren $R$, Sivapathasundharam B. Benign and malignant tumour of oral cavity. In: Shafer textbook of oral pathology. $5^{\text {th }}$ edition. New Delhi: Reed Elsevier India Pvt limited;2005.239-244.

8. Mirra JM, (ed). Bone Tumors: Clinical, Radiologic and PathologCorrelations. Philadelphia: Lea and Febiger: 1989;250-252.

9. Abraham LA, Yamane GM, Glickman RS, Stuart Super, Salman L, Chaudhary AP. Chondroblastic osteosarcoma of the mandible 10 years after a squamous cell carcinoma of the contralateral side (report of a case). Journal of Oral Medicine. 1986;41:4-8.

10. Nissanka EH, Amaratunge EAPD, Tilakaratne WM. Clinicopathological analysis of osteosarcomaof jaw bones. Oral disease. 2006; 13:82-87.

11. Sainin R, Razak NHA, Rahman SA, Samsudin AR. Chondrosarcoma of the mandible: a case report. JCDA. 2007; 73:175-178.

12. Conventry $M B$, Dahlin DC. Osteogenic sarcoma: a critical analysis of 430 cases. JB \& JS. 1957;39:741-758. 